\title{
SISTEMA DE GESTÃo DE ARTEFATOS PEDAGÓGICOS
}

\author{
Lucas H. A. Flaquer e Johannes V. Lochter \\ Departamento da Computação \\ Centro Universitário Facens \\ Sorocaba/SP - Brasil
}

\section{RESUMO}

As tecnologias de informação vêm ganhando cada vez mais importância na área de educação. Mesmo que artefatos pedagógicos, como planos de ensino e planos de aula, não sejam feitos à mão, os processos de digitalização e desenvolvimento, aprovação e divulgação para os alunos no início do semestre, ainda são feitos sem apoio computacional, o que gera certa sobrecarga em professores que ministram diversas disciplinas e têm de fazer este processo antes do início do período letivo. O presente trabalho propõe acelerar esse processo, tornando-o dinâmico e menos propenso a erros com uma aplicação web para gerenciar planos de aula de uma instituição de ensino. A partir dessa aplicação de código aberto, espera-se produzir um meio eficaz de automatizar processos evitando erros e atrasos.

\section{PALAVRAS-CHAVE}

Aplicação Web, Artefatos Pedagógicos, Computação Aplicada

\section{INTRODUÇÃ̃}

O mundo todo passa por uma transformação de dados irreversível. As pessoas consomem cada vez mais eletrônicos que registram e geram informações em nuvens tecnológicas capazes de armazenar infinidade de dados. As indústrias também passaram a aderir a essa transformação com a Indústria 4.0, assim como o agronegócio e todo o setor educacional (Lempinen, 2020).

Em 2020, uma crise de saúde mundial resultante de um vírus apelidado de Covid-19, transformou a vida de todos habitantes do planeta ao colocá-los em confinamento para mitigar a contaminação da população, afetando a forma como as pessoas trabalham e estudam. Muitas empresas se viram obrigadas a colocar seus funcionários em regime de trabalho home-office, enquanto muitas instituições de ensino tiveram que procurar alternativa para as aulas presenciais que não poderiam acontecer devido às restrições dos governos seguindo recomendações da Organização Mundial de Saúde.

Entre as alternativas para as aulas presenciais, as instituições passaram a adotar aulas telepresenciais durante o confinamento, nas quais o professor utiliza o mesmo horário das aulas presenciais para ter um momento síncrono com os alunos utilizando alguma ferramenta de videoconferência. Exemplo disso foi a alavancagem das ações da empresa Zoom (Figura 1), cuja ferramenta de mesmo nome foi uma das mais utilizadas no momento da pandemia.

As mudanças tecnológicas no setor educacional foram marcadas por muito mais tecnologia que a adaptação de uma ferramenta de videoconferência para possibilitar aulas telepresenciais para os alunos. Principalmente no ensino superior, o setor já usa há anos ferramentas para fazer gestão dos estudantes, como matrículas e grade curricular, e ferramentas voltadas para aprendizagem, como disponibilização de conteúdo em portais, gestão de notas e faltas. Alguns dos sistemas de aprendizagem apresentam soluções avançadas, como implementações de aprendizagem adaptativa, as quais utilizam inteligência artificial para recomendar conteúdo de acordo com a dificuldade de cada estudante (Basitere \& Ivala, 2017).

Os sistemas que fazem a gestão do cadastro dos estudantes, professores, estoque de materiais, controle de matrículas, recebimentos e contas a pagar nas instituições de ensino, normalmente são sistemas de gestão empresariais (ERP, em inglês). Esses sistemas tratam a instituição de ensino como uma empresa de qualquer outro setor que tem necessidades em comuns, tanto contábeis, como administrativas e recursos humanos (Kokate, 2014; Abugabah \& Louis, 2010). 


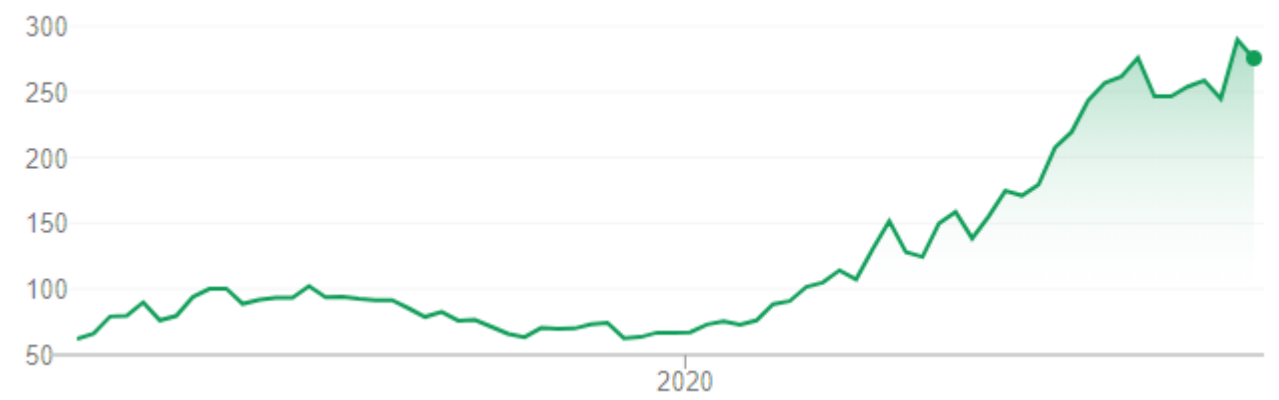

Figura 1. Gráfico de evolução das ações da empresa Zoom

Fonte: https://www.istoedinheiro.com.br/no-foco-do-zoom/, acessado em 05/08/2020

Este trabalho propõe uma ferramenta chamada Artefactus para catalogar e gerenciar os artefatos pedagógicos pertences aos cursos de ensino superior, como as grades curriculares e seus componentes, bem como competências e objetivos de aprendizagem. Essa ferramenta tem distribuição livre sem custos, possui interface web para fácil acesso e busca promover a simplicidade construída sobre uma base de código robusta. O restante deste trabalho está organizado da seguinte maneira: os aspectos teóricos são apresentados na Seção 2; a metodologia e o desenvolvimento na Seção 3; na Seção 4 são apresentados os resultados e as conclusões são discutidas na Seção 5 .

\section{FUNDAMENTAÇÃO TEÓRICA}

Sistemas de gestão educacional são bem comuns e se tornaram populares com o aumento massivo de procura por cursos profissionalizantes, demandando esforço e processos digitais para atender ao maior número de alunos. Esses sistemas são facilmente confundidos pelas diferentes vertentes que se encaixam, como sistemas que auxiliam a vida do aluno e sistemas que auxiliam a gestão da própria instituição, como contábil, pedagógico e financeiro.

Os sistemas que facilitam a vida do aluno incluem acesso a notas, conteúdo das aulas, registros de faltas e, normalmente, são utilizados como um importante canal de comunicação entre a instituição e o aluno. Para Araújo Júnior \& Marquesi (2009), esses sistemas permitem a comunicação entre instrutor e aluno de maneira síncrona e assíncrona, permitindo utilizar várias estratégias para engajamento dos alunos. Do ponto de vista de Lonn \& Teasley (2009), esses sistemas são baseados na interface web e específicos para compartilhar materiais, enviar tarefas e ter acesso a feedback com notas.

$\mathrm{Na}$ instituição de ensino, os sistemas de gestão ajudam na manutenção financeira e contábil, além de permitir o controle dos assuntos pedagógicos relacionados ao aluno, como matrícula, aproveitamento e emissão de certificados. As instituições normalmente dependem de ambos os sistemas para oferecer facilidades digitais aos alunos, além de ter seus próprios processos em formato digital. No entanto, não é comum que esses sistemas façam a gestão dos planos pedagógicos de curso e que tenham algum tipo de registro e lastro dos objetos de aprendizagem que permeiam a formação curricular dos alunos.

A gestão do plano pedagógico do curso é importante principalmente porque há auditorias recorrentes de órgãos regulamentadores, como associações de uma categoria específica ou o Ministério da Educação e Cultura, a tomar o Brasil como exemplo, as quais buscam averiguar se a instituição de ensino continua ofertando cursos que atendam requisitos mínimos estabelecidos.

O plano pedagógico do curso contempla, dentre outras coisas, as disciplinas, competências do curso e os objetivos de aprendizagem. As disciplinas de um curso devem atender competências esperadas desses órgãos reguladores. Essas competências podem ser separadas em objetivos de aprendizagem propostos para cada disciplina. Um exemplo é a disciplina de algoritmos do curso de Engenharia de Computação que deve atender à regulação da Diretriz Curricular Nacional proposta pelo Ministério da Educação e Cultura no Brasil, além de observar também as diretrizes dadas pelo Conselho Federal de Engenharia e Agronomia, que regulamenta as engenharias. Nessa disciplina, um exemplo de competência é "Conhecer, entender e aplicar conceitos de algoritmos para projetar sistemas inteligentes”. Nessa competência, a disciplina explora objetivos de 
aprendizagem como "Entender e aplicar laços de repetição para construir aplicações capazes de processar dados de maneira iterativa".

As competências e os objetivos de aprendizagem são partes essenciais e integrantes do plano pedagógico do curso e não há registros na literatura de soluções tecnológicas que organizem esses artefatos pedagógicos de maneira que a consulta seja simples, bem como o cadastro e a gestão, pelos coordenadores pedagógicos, coordenadores de curso, professores e alunos da instituição.

Esse trabalho propõe um sistema para o fim de organizar e permitir a gestão eficiente desses artefatos pedagógicos, desenvolvido com boas práticas, permitindo personalização do ambiente para atender os diversos fins de cada instituição, além de oferecer as funcionalidades básicas que atendam o objetivo proposto.

\section{METODOLOGIA E DESENVOLVIMENTO}

Para permitir a construção do sistema Artefactus, foram escolhidas tecnologias populares e recentes para a arquitetura (Figura 2). O sistema utiliza interface web no modelo cliente-servidor onde, o cliente é responsável por fazer o envio de requisições com as informações salvas pelo usuário, como cadastro de planos de aula, alterações de permissões de acordo com o tipo do usuário, dentre outras.

O servidor por sua vez, tem a responsabilidade de receber as requisições e envios de dados do lado do cliente, realizar validações de segurança de acordo com as regras de negócio, armazenar os dados válidos no banco de dados e realizar requisições no mesmo para disponibilizar os dados para o cliente. A comunicação entre o cliente e o servidor é feita no formato de API (Application Programming Interface) que utiliza requisições HTTP organizadas pelos verbos HTTP GET, POST, PUT/PATCH e DELETE e validados utilizando um token de autenticação chamado JWT (JWT2018).

No lado do cliente foi utilizado o framework Javascript Vue.js (2018) para o desenvolvimento das telas. É um framework simples e adaptável. Para o controle de rotas, foi usado Vue Router (2018), o qual se integra ao núcleo do Vue.js para tornar a criação de aplicativos de página única com Vue.js simples. Também foi utilizado o padrão de gerenciamento de estado centralizando todos os dados com regras garantido que só possam ser alterados a partir de regras e formas previsíveis chamado de Vuex (2018).

No lado do Servidor foi utilizado para desenvolvimento a linguagem Python em sua versão 3.8 e o framework web Flask (2020) junto da biblioteca Flask-Restful (2020) para o desenvolvimento da API.

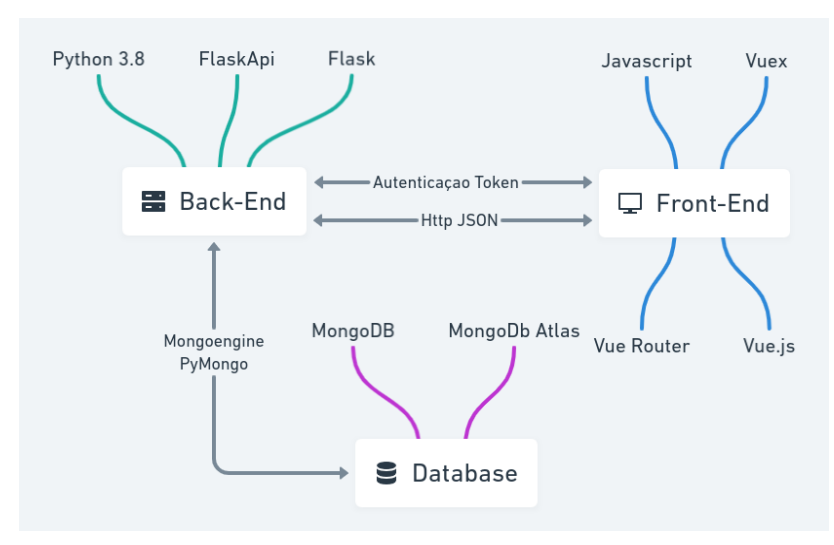

Figura 2. Arquitetura utilizada

Fonte: Elaborado pelos autores

Foi utilizada uma arquitetura em camadas onde tem-se a camada de rotas que gerencia as rotas do sistema; a camada de controle que recebe os dados das rotas e realiza validações e comunica com a camada de serviço; a camada de serviço faz a comunicação com o banco de dados consultando os modelos definidos na camada de modelo para retornar os objetos correspondentes as requisições.

O banco de dados utilizado foi o MongoDB (2020) que tem sua estrutura baseada em documentos, ou seja, ele é um banco de dados não relacional. A base de dados de testes está sendo fornecida através de um serviço chamado MongoDB Atlas que fornece um cluster de até 500MB sendo salvos em nuvem. 


\section{RESULTADOS}

O sistema Artefactus propõe fornecer os meios para facilitar a gestão dos artefatos pedagógicos da instituição de ensino utilizando diferentes papeis e permissões que caracterizam diferentes perfis dentro do sistema. Por meio dos diferentes perfis, cada usuário também se torna responsável por diferentes ações. Assim, os usuários assumem responsabilidades diferentes quanto à criação de cursos, disciplinas, ementas e até mesmo do nível mais granular, que é a própria aula.

Conforme ilustrado pelo diagrama de casos de uso (Figura 3), caberá ao professor consultar as disciplinas que ministra e realizar o cadastro ou edição dos planos de aula das disciplinas que ministra no semestre. $\mathrm{O}$ professor NDE (núcleo docente estruturante) é um perfil de acesso mais alto que os professores, porém inferior ao coordenador do curso, que permite gerenciar as atividades dos outros professores, com a elaboração das ementas. Por fim o coordenador possui o papel de gerenciar as disciplinas do curso podendo modificar a grade do curso que ministra, adicionar novos usuários e designar papéis aos mesmos, estendendo as funcionalidades do professor NDE para todas as disciplinas do curso que coordena e executando também as mesmas funcionalidades do professor comum como o cadastro de planos de aula. Na Figura 4, é possível adicionar um novo plano de aula e vincular informações a este.

Este é um projeto de código aberto e está disponibilizado para qualquer um que queira usar ou contribuir através destes dois repositórios no Github: https://github.com/LucasFlaquer/plano-aula e https://github.com/LucasFlaquer/plano-aula-api.

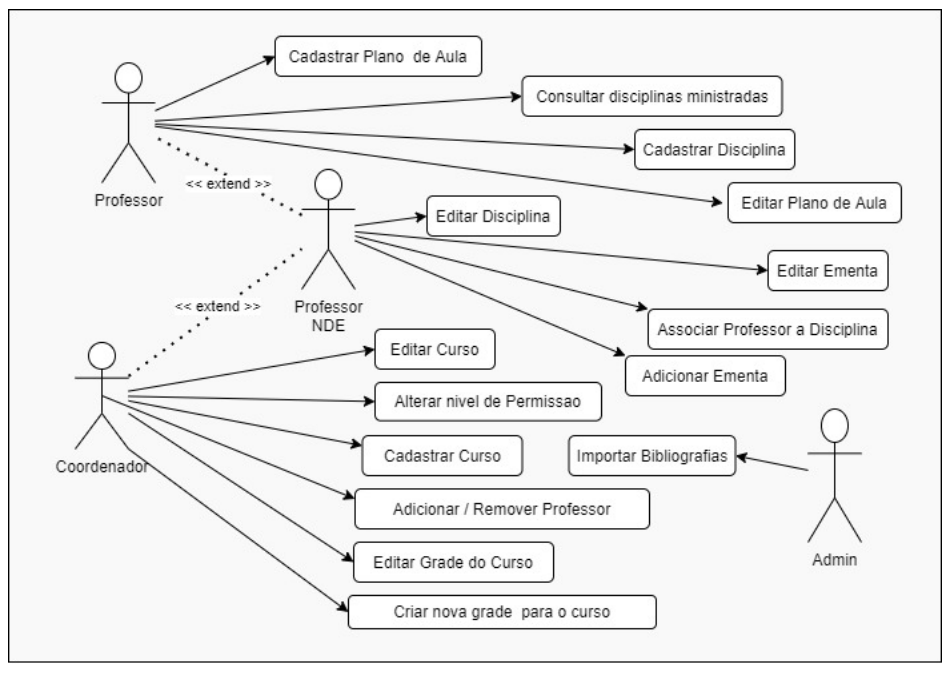

Figura 3. Diagrama de casos de uso Fonte: Elaborado pelos autores

\section{CONCLUSÃO E TRABALHOS FUTUROS}

As instituições de ensino carecem de sistemas de gestão que não se preocupam apenas com os aspectos empresariais, como estoque e contas a pagar, mas também com sistemas que gerenciem os artefatos pedagógicos para simplificar, por exemplo, a auditoria de órgãos reguladores de educação.

Este trabalho traz a proposta do sistema Artefactus para gerenciar artefatos pedagógicos dentro da instituição com toda estrutura para inclusão de cursos, corpo docente, permissões de escrita e leitura, integração com referências bibliográficas.

Os próximos passos do desenvolvimento incluem melhorias visuais na interface, área de cadastro de novos professores com as devidas parametrizações no banco de dados, implementação de ambiente de homologação com professores alimentando com dados reais a aplicação e por fim, a publicação e utilização por todos os professores do corpo docente. 


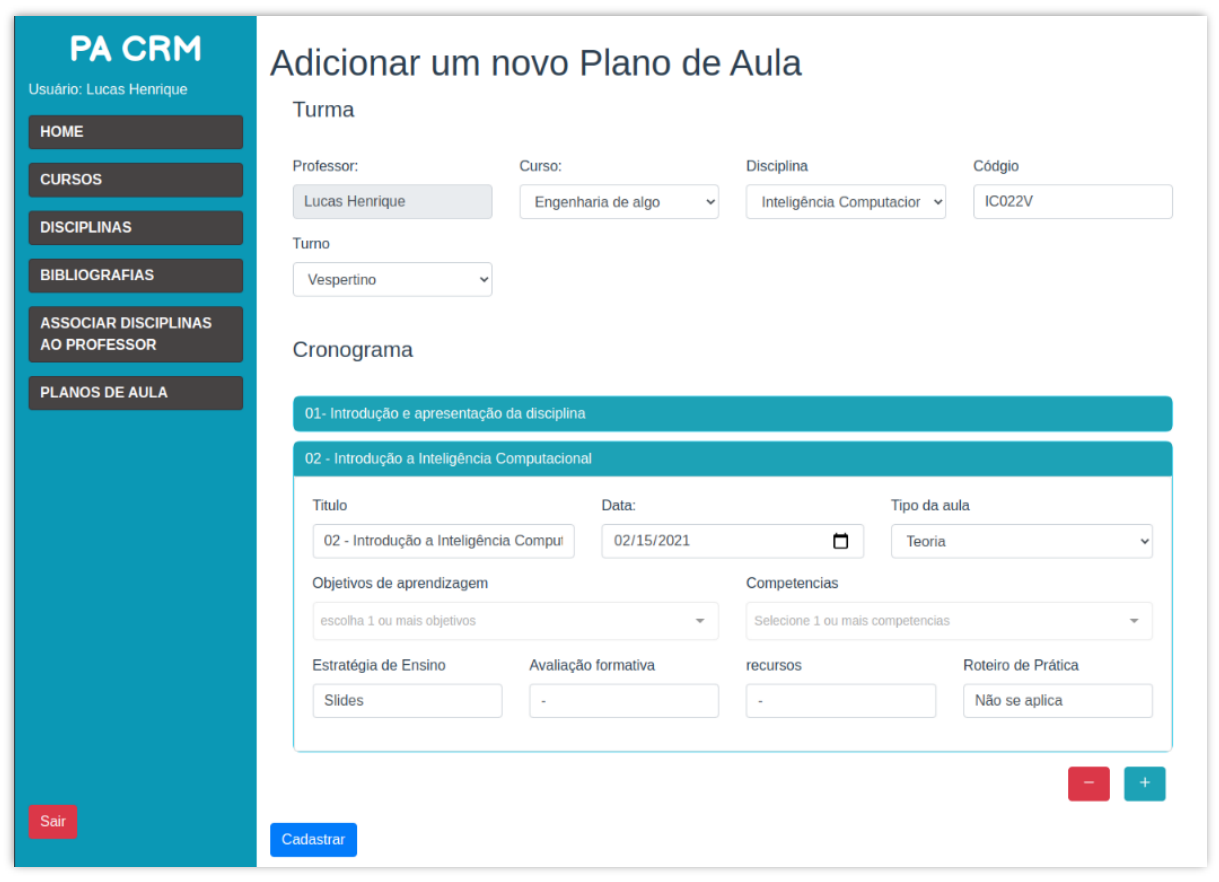

Figura 4. Tela de Cadastrar plano de aula Fonte: Elaborado pelos autores

\section{REFERÊNCIAS}

Abugabah, Ahed \& Sanzogni, Louis, 2010. Enterprise Resource Planning (ERP) System in Higher Education: A literature Review and Implications. 71.

Araújo-Junior, C.F., \& Marquesi, S.C., 2009. Atividades em ambientes virtuais de aprendizagem: parâmetros de qualidade. In: LITTO, F.M., \& Formiga, M. (Eds.), Educação a distância: o estado da arte. Pearson, São Paulo. pp. 358-368.

Basitere, Moses \& Ivala, Eunice, 2017. Evaluation of an adaptive learning technology in a first-year extended curriculum programme physics course. South African Computer Journal, 29(3), 1-15.

Edward Lempinen, 2020. The pandemic could open a door to new technology — and dramatic innovation — in education. Disponível em https://news.berkeley.edu/2020/05/27/the-pandemic-could-open-a-door-to-new-technology-anddramatic-innovation-in-education/, acessado em 29/09/2020.

FLASK JWT, 2018. Disponível em https://flask-jwt-extended.readthedocs.io/, acessado em 10/08/2020.

Flask Restful, 2020. Disponível em https://flask-restful.readthedocs.io/en/latest/, acessado em 25/09/2020.

Flask, 2020. Disponível em https://flask.palletsprojects.com/en/1.1.x/, acessado em 25/09/2020.

JWT, 2018. Disponível em https://jwt.io/introduction/, acessado em 23/09/2020.

Kokate, Shrikant, 2014. E-College: An ERP for Educational Institute, em International Journal of Computer Science and Engineering.

Lonn, S., \& Teasley, S., 2009. Saving time or innovating practice: investigating perceptions and uses of learning management systems. Computers \& Education, 53(3), 686-694.

Mongo DB, 2020. Disponível em https://www.mongodb.com/, acessado em 25/09/2020.

Vue Router, 2018. Disponível em https://router.vuejs.org/, acessado em 25/09/2020.

Vue, 2018. Disponível em https://vuejs.org/, acessado em 23/09/2020.

Vuex, 2018. Disponível em https://vuex.vuejs.org/, acessado em 25/09/2020. 